\title{
Jean-Luc Godard : autoportrait(s) d'un cinéaste
}

\section{Didier Coureau}

\section{OpenEdition}

Journals

Édition électronique

URL : http://journals.openedition.org/recherchestravaux/382

ISSN : 1969-6434

\section{Éditeur}

UGA Éditions/Université Grenoble Alpes

Édition imprimée

Date de publication : 30 décembre 2009

Pagination : 111-122

ISBN : 978-2-84310-159-5

ISSN : 0151-1874

\section{Référence électronique}

Didier Coureau, « Jean-Luc Godard : autoportrait(s) d'un cinéaste », Recherches \& Travaux [En ligne],

75 | 2009, mis en ligne le 30 juin 2011, consulté le 07 septembre 2020. URL : http://

journals.openedition.org/recherchestravaux/382 


\section{Jean-Luc Godard : autoportrait(s) d'un cinéaste}

Autoportrait(s) d'un cinéaste au travail, dans l'acte de création, ou à côté, ou en deçà, ou au-delà. Autoportrait(s) filmique(s) qui démultiplie(nt) les images de soi - attitudes et postures alliées aux flux de la pensée -, et de l'image de l'autre en soi - dédoublements et masques : JLG/JLG. Autoportrait(s) fragmentaire(s) ou cristallin(s) - ainsi que dans l'Image-Temps de Gilles Deleuze. Non plus la "glace à trois faces» du film de Jean Epstein, mais une multiplicité de facettes qui renvoient fragments d'images et éclats de voix, faisant miroiter les virtualités de l'histoire : d'un homme, d'un personnage (conceptuel), de l'art, du monde. Autoportrait(s) complexe(s) : interactions de l'Un et du pluriel, dans une esthétique qui répond, en ces incessantes métamorphoses, à l'intitulé de cette réalisation majeure de Jean-Luc Godard : Histoire(s) du cinéma.

\section{Autoportrait cinématographique}

En 1994, il est proposé à Godard de réaliser son autoportrait, qui sera diffusé en concomitance avec la célébration du premier siècle d'existence du cinématographe des frères Lumière, l'année suivante. L'intitulé choisi par le cinéaste est significatif, puisqu'il affirme sa volonté de ne pas faire un "JeanLuc Godard par Jean-Luc Godard », mais d'utiliser son nom "codé», "JLG», en le redoublant de part et d'autre d'une barre oblique. Le titre devient ainsi $J L G / J L G$, et est assorti d'un sous-titre, "autoportrait de décembre», mois qui est celui de sa naissance (tandis que décembre I895 est le mois officiel de celle du Cinématographe). Bien sûr, une telle réalisation s'accompagne d'une 
véritable réflexion sur la notion même d'autoportrait. Dans un premier temps, Godard a souhaité marquer la différence entre autoportrait et autobiographie, et a déclaré en ce sens :

$J L G / J L G$ n'est pas une autobiographie, mais un autoportrait, au sens où des peintres ont pratiqué cet exercice : non par narcissisme, mais comme une interrogation sur la peinture elle-même, et leur possibilité de s'y fondre. Cette démarche fait partie de la conception occidentale de l'art : l'art est plus grand que les hommes, plus grand que les artistes. [...] Moi, je considère toujours le cinéma comme plus grand que moi, JLG/JLG est un essai pour voir ce que le cinéma peut faire avec moi, pas ce que je peux faire de lui'.

Puis, il a précisé la distinction qui peut être effectuée entre les domaines littéraire et pictural :

C'est un genre très courant en peinture. Pas tellement en littérature - il y a des autobiographies, des souvenirs, des mémoires et même des mémoires d'outretombe. [...] j’ai tenté de comprendre ce que pouvait être un autoportrait cinématographique. Jusqu'où je pouvais aller dans le cinéma et jusqu'où le cinéma pouvait m'accepter ${ }^{2}$.

Enfin, il a souligné le défi qu’il lui semblait devoir relever afin de créer cet autoportrait cinématographique : "JLG/JLG est un autoportrait, chose qui me semble impensable à faire au cinéma, mais le cinéma est fait pour penser l'impensable $[\ldots]^{3}$.»

La comparaison menée par Godard porte, de manière logique, sur la peinture, même s'il a également pu faire allusion, à propos de son film, à des sources littéraires à portée philosophique, Maurice Blanchot - penseur de l'écriture et de l'«exigence fragmentaire» -, et Georges Bataille auteur de L'Expérience intérieure.

Mais il s'agit surtout de penser cette «impensable» notion d'autoportrait cinématographique, en insistant sur ses spécificités, et de montrer que cette interrogation est très antérieure à $J L G / J L G$, puisqu'elle parcourt en fait toute la filmographie du cinéaste. Les exemples pris, qui couvrent la vaste période 1958I998, viseront à le prouver. Quarante années, telle fut aussi la durée pendant laquelle Rembrandt se représenta inlassablement dans sa peinture. Godard écrit justement du peintre hollandais - déjà cité dans Passion, où était reconstituée sous forme de tableau vivant $L a$ Ronde de nuit -, au sein d'une version du scénario de son film : «Mais que cherchait[-t-il] lorsqu'il installa le chevalet

I. J.-L. Godard, dans J-M. Frodon, "JLG/NYC (Jean-Luc Godard au musée d'Art moderne de New York), Le Monde, mardi io mai i994, p. I9.

2. Id., dans "Une bouleversante expérience intérieure", propos recueillis par I. Danel, Télérama, $\mathrm{n}^{\circ} 2356,8$ mars $\mathrm{i} 995$, p. 30

3. Id., dans Jean-Luc Godard par Jean-Luc Godard, t. 2, A. Bergala éd., Cahiers du cinéma, I998, p. 294. 
à côté du miroir. Probablement : jusqu'où la peinture peut aller4. » De Godard, comme de Rembrandt, sont ainsi connus les visages de leurs âges successifs, comme en un feuilletage mémoriel qui conserverait les multiples strates qui composent l'être. Mais n'est-ce pas justement déjà l'aveu fait d'une impossible unité de soi, tant toutes ses représentations ne donnent que des facettes fragmentaires de l'individu, saisi dans des instantanés au fil du temps?

Dès $\grave{A}$ bout de souffle (1960), Godard apparait de manière brève et symbolique dans son film. En cette période de la "politique des auteurs" énoncée par les rédacteurs des Cahiers du cinéma, qui deviendront peu à peu les cinéastes de la Nouvelle Vague, l'influence d'Hitchcock qui aimait inscrire sa silhouette dans ses films est manifeste. Godard ne se donne pas n'importe quel «rôle», puisqu'il interprète celui d'un délateur. Dénoncer, en tant que cinéaste, son personnage Michel Poicard, c'est peut-être aussi dénoncer plus largement la fiction en son ensemble, et par là même la continuité narrative du récit. Godard est d'abord entendu sans être vu, pour être ensuite vu sans être entendu, et être finalement cerné par une fermeture à l'iris, hommage ironique au cinéma muet (comme aimait également en faire François Truffaut). Un rapport voir/entendre particulier se noue déjà dans ces plans, puisqu'un autoportrait cinématographique passe, évidemment, par des dimensions sonores et visuelles... Cette apparition du créateur dans son œuvre se fait dans un montage bien particulier, caractérisé par une certaine complexité, que l'on a de manière un peu simplificatrice associé à la simple idée de faux raccord, mais qui implique les idées de fragmentation et de discontinuité.

\section{Autoportrait fragmentaire}

Dans Pierrot le fou (1965), le personnage de Jean-Paul Belmondo (qui porte $\mathrm{du}$ reste deux prénoms selon ses interlocuteurs : Pierrot et Ferdinand) déclare :

J'ai une machine pour voir qui s'appelle les yeux; pour entendre : les oreilles; pour parler : la bouche. J'ai l'impression que c'est des machines séparées. Y'a pas d'unité. Je devrais avoir l'impression d'être unique, j'ai l'impression d'être plusieurs.

À l'idée d'autoportrait s'adjoignent alors clairement celles de la représentation fragmentaire au niveau de la disjonction entre voir, entendre, dire, et de la démultiplication de l'être... Pistes qui peuvent éclairer l'ensemble de la démarche de Godard, comme cela se remarque par exemple dans certains

4. Ibid., p. 286. 
passages de trois de ses films : Charlotte et son Jules, Vivre sa vie, Sauve qui peut (la vie).

Dans le court métrage Charlotte et son Jules (1958) - l'une de ces esquisses immédiatement antérieures à $A$ bout de souffle -, Belmondo (prénommé Jean, dans le script, soit la moitié des prénoms composés Jean-Paul et JeanLuc) est vu, mais c'est Godard qui le postsynchronise en lui prêtant sa voix. Lautoportrait se fait alors avant tout sonore, bien que dans le personnage masculin, scénariste ou cinéaste manqué, qui prononce quelques paroles définitives sur l'absurdité du cinéma, ce spectacle dérisoire où des "grosses têtes font des grimaces sur un petit écran ", se reconnaissent l'ironie acerbe et l'autodérision de Godard. Jean-Paul Belmondo est ici un personnage qui remue les lèvres comme au temps du cinéma muet, tandis que le cinéaste le fait parler - comme un bonimenteur le faisait parfois, caché derrière l'écran, dans les premiers temps du cinéma -, tandis que Charlotte (Anne Colette) mime Jean au second plan, et se dédouble elle-même dans une projection de son ombre sur le mur, tout cela proposant, sous l'apparente légèreté, une réflexion historique sur un art qui commença dans la disjonction du corps et de la voix.

Dans Vivre sa vie (196I), Godard réitère l'expérience, et prête cette fois sa voix à Peter Kassovitz (cinéaste pour l'occasion comédien muet). Mais il prend simultanément à son compte la nouvelle d'Edgar Poe, Le Miroir ovale, dans la célèbre traduction de Charles Baudelaire, afin de faire le portrait d'Anna Karina, ici nommée Nana en hommage à l'adaptation de Zola par Jean Renoir. La séquence, qui commence et se termine par des plans intégrant des sous-titres - dans l'idée des cartons de dialogues du cinéma muet -, cède ensuite la place à cette lecture faite par la voix de Godard, et aux gros plans sur le visage de Anna Karina. Le cinéaste introduit au milieu de la séquence la réplique : "C'est notre histoire, un peintre qui fait le portrait de sa femme», qui vient montrer la fusion entre le texte de Poe et sa propre pensée, mais avec le paradoxe de venir habiter le corps d'un comédien pour faire cette déclaration. Ce nouvel autoportrait vocal est aussi de l'ordre d'une confession, qui révèle la conscience du cinéaste d'être un portraitiste "vampirique» de sa comédienne qui n'est autre que la femme aimée... Car, comme on le sait, la nouvelle fantastique de Poe évoque la vie que la peinture et le portrait peint viennent puiser dans le modèle de chair et de sang. Jean Epstein en donna une mémorable représentation impressionniste dans son film muet La Chute de la maison Usher (1928).

Dans Sauve qui peut (la vie) I979 - où se sent une certaine analogie avec le titre du film précédent -, n'apparaissent ni l'image de Godard ni sa voix, mais son nom de famille rattaché à un autre prénom, Paul, et au comédien Jacques Dutronc. Paul Godard se retrouve dans une séquence face à un 
JeAn-Luc Godard : Autoportrait(s) D'un CinÉaste

groupe d'étudiantes, et est censé accueillir Marguerite Duras. Celle-ci n'a en réalité pas accepté la proposition faite par Godard d'apparaître à l'écran, et son refus est intégré au film. Paul Godard, à défaut de la recevoir, se contente de la citer :

Je fais des films pour occuper mon temps. Si j'avais la force de ne rien faire, je ne ferais rien. C'est parce que je n'ai pas la force de ne m'occuper à rien que je fais des films. Pour aucune autre raison. C'est là le plus vrai de tout ce que je peux dire sur mon entreprise,

et de dire son adhésion à ses propos. Il est alors légitime de se demander quel est le «je» qui parle, et il est aisé de penser que cette affirmation ironique, empreinte de doute, sied à Godard (Jean-Luc). L'autoportrait se fait de manière détournée, indirecte, à travers le corps d'un autre, mais qui tient le rôle d'un cinéaste qui, à ce moment précis, rend hommage à Marguerite Duras qui ne sera présente que de manière vocale - voix off sur un travelling mémorable à travers Lausanne - dans le film, comme Godard le fut parfois dans certains de ses films.

\section{Je est un autre}

Godard oscille dans les trois cas précédents entre le "Je suis l'autre" de Gérard de Nerval, affirmation d'une sévère crise identitaire, et le "Je est un autre» d'Arthur Rimbaud, qui peut être considéré comme une ouverture à la multiplicité. Dans les trois extraits, la présence-absence de Godard lui-même dénonce évidemment l'unicité de l'autoportrait, pour en faire une sorte d'autoportrait en "Je est partiellement un autre» (présence sonore, présence par le nom), ou en "Je» recomposé. Le vers de Rimbaud apparaît dans un carton-citation d'un autre film, Nouvelle Vague (1990) - film sur le dédoublement -, et a toujours été au cœur des réflexions du cinéaste qui se sent souvent étranger à lui-même, comme il a pu le préciser au moment de $J L G /$ $J L G$ : "Tout à coup vous vous apercevez que vous êtes un autre. Alors si Je est un autre, quel est Je? Et qui est cet autres.» Il lui est aussi arrivé de mentionner en ce sens Emmanuel Levinas, comme dans For Ever Mozart (1996) : «dans je pense donc je suis, le je du je suis, n'est plus le même que le je du je pense, parce qu'il reste à démontrer qu'il y a un rapport entre le corps et l'esprit, entre pensée et existence». Le philosophe Léon Brunschvicg, qui affirme selon Godard que "L'un est dans l'autre et l'autre est dans l'un, et ce sont les trois personnes", étant également souvent cité par le cinéaste,

5. J.-L. Godard, dans "Comme un poisson dans l'eau", propos recueillis par M.-E. Rouchy, Télérama, $\mathrm{n}^{\circ}$ 2107, 30 mai 1990, p. 29. 
comme une réponse à l'énigme du dédoublement. Cette phrase apparaît, par exemple, de manière fragmentaire dans une succession de cartons au sein de Hélas pour moi (1993) : "Tout est dans un / Et l'autre / Et l'autre est / Et l'autre est dans / Et l'autre est dans un / Et ce sont / Et ce sont les trois personnes.»

Mais Godard est aussi, bien évidemment, présent de manière à la fois visuelle et sonore dans d'autres de ses films, au-delà de l'exemple mentionné d'À bout de souffle, ce qui ne garantit pas pour autant l'idée d'une unification possible. Il est ainsi possible de proposer un petit parcours chronologique dans sa filmographie, et de citer quelques moments où la présence de l'autoportrait se fait manifeste.

\section{Lignes}

Lorsqu'il est proposé à Godard de participer au film collectif militant Loin du Vietnam (1967) - initié par Chris Marker -, celui-ci réalise un film où il se met en scène, manière paradoxale de répondre à un projet où les participants ne souhaitent pas signer leur film, en vertu de leur engagement politique commun. C'est la première fois que Godard fait son autoportrait en cinéaste au travail. Cependant, c'est là aussi que les choses se complexifient. Le titre Caméra-Eil de son court métrage renvoie évidemment au grand cinéaste et théoricien soviétique Dziga Vertov, à son «Kino-Glaz» ("Ciné-CEil»), dont il écrivait : "Je suis le ciné-œil. Je suis l'œil mécanique. Moi, machine, je vous montre le monde tel que moi seul peut le voir ${ }^{6}$.» Godard est ici l'Homme à la caméra, selon le titre célèbre du film de Vertov, et même pourrait-on dire l'homme-caméra tant il fusionne avec elle ${ }^{7}$, sur cette terrasse d'immeuble parisien, depuis laquelle il semble vouloir capter tous les soubresauts de l'humanité. Cependant, plus que ce qui est vu, importe ce qui est entendu : la voix de Godard et une autre voix off, dans l'entrecroisement de deux récits; et les bruits de la guerre lointaine qui se font entendre ici et maintenant. «Il faut que l'œil écoute avant de regarder» est une phrase entendue dans Le Gai savoir de Godard en 1968, allusion faite au titre d'un ouvrage de Paul Claudel consacré à la peinture : L'ail écoute. Godard précisait d'ailleurs au moment

6. D. Vertov, cité dans G. Sadoul, Dziga Vertov, Champ libre, 197I, p. 9I.

7. Un film non réalisé de Godard, en I973, devait s'intituler Moi je et, s'appuyant sur Différence et répétition de G. Deleuze, comporter deux parties : "Moi, je suis un homme politique" et "Moi, je suis une machine». Au moment de $J L G / J L G$, le cinéaste évoquait la présence d'un JLG "devant la caméra», et de «l'autre dedans» (J.-L. Godard, dans "Une bouleversante expérience intérieure", op. cit.). 
de la sortie de Sauve qui peut (la vie) : "Ce qui m'intéresse [...] c'est de voir ce qu'on entend et d'entendre ce qu'on voit ${ }^{8}$.» Ce qui passe dans cette image d'un homme à la caméra - comme un peintre avec son pinceau -, c'est la multiplicité des «lignes» qui traversent un individu selon Gilles Deleuze : lignes sociales à segments durs, lignes souples des flux et des devenirs, lignes de fuites créatrices ou destructrices. Et ce sont ces lignes, sous leurs formes sonores et visuelles, qui composent l'autoportrait fragmentaire de Godard, celle d'un créateur parcouru par tous les bruits du monde...

En 1982, dans Lettre à Freddy Buache, la bande-son est encore primordiale, mais un élément nouveau est mis au premier plan : la main qui met en marche les outils de la production audiovisuelle et, plus particulièrement, ceux de l'enregistrement sonore... "Penser avec les mains", titre du philosophe Denis de Rougemont, est une formule devenue obsessionnelle pour Godard. C'est par la main que passe le son. Ce qui est également montré, après avoir été explicité par la voix off qui donne à entendre une conversation entre Godard et la police, évoque en fait l'absence d'un plan qui ne pourra jamais être capté, un moment de lumière à saisir sur la bande d'arrêt d'urgence de l'autoroute. Est alors vu - et entendu dans une certaine désynchronisation -, à travers un disque 33 tours sur un électrophone, le Boléro de Maurice Ravel, relié aux mains de Godard au premier plan, son visage, de face, restant partiellement voilé dans l'ombre. Double circularité de la musique et du disque, qui fait des boucles et réunit les lignes fragmentaires et multiples de cet autoportrait du cinéaste en amateur de musique...

\section{Personnages conceptuels}

Un passage de Prénom, Carmen (1983) peut faire songer à une réinterprétation des éléments en présence dans le court métrage de Loin du Vietnam. Mais, ici, la caméra-radio prend la forme d'un véritable poste de radio : y passent les notes égrenées de Mon ami Pierrot (allusion évidente à Pierrot le fou), et les bruits de la guerre (extérieure ou intérieure à Godard). Quant au Quatuor à cordes, également présent dans le film, il est entendu lors des répétitions de l'une des dernières œuvres de Beethoven, en lieu et place de la musique de Bizet que le titre pouvait laisser attendre.

Mais, ce qui apparaît aussi dans Prénom, Carmen, et sous une autre forme dans Soigne ta droite (1987), c'est ce que Deleuze et Guattari nomment des "personnages conceptuels»: Godard/Oncle Jean dans Prénom, Carmen;

8. J.-L. Godard, cité dans J.-L. Douin, Jean-Luc Godard, Rivage, "cinéma», I989, p. 98. 
et le double personnage Godard/L'Idiot - Godard/Le Prince dans Soigne ta droite qui débouche sur ce troisième personnage : Godard/L'extraterrestre qui, selon Godard, "a été mis sur la terre [...] [et] qu'il faut découvrir, soit comme espion, soit comme fiction'» (Soigne ta droite est d'ailleurs sous-titré "Une place sur la terre»). Oncle Jean et L'Idiot sont, en définitive, presque le même "personnage conceptuel». Deleuze et Guattari évoquent l'Idiot de Descartes, "penseur privéro " qui accède à la raison; mais aussi l'Idiot de Dostoïevski, un "Descartes en Russie devenu fou ${ }^{\mathrm{II}}$ ", qui ne peut plus que vivre dans l'absurde. C'est à ce dernier que Godard fait allusion à travers la couverture du livre de la collection Folio de Gallimard, très nettement visible et lisible, sur laquelle est inscrit «L'Idiot 2 ». Titre qui contient le chiffre deux, évidemment symbolique du dédoublement, mais peut également faire référence, de manière ironique et critique, aux films commerciaux à épisodes souvent dénoncés par Godard... Dans les deux films, le cinéaste est à la fois une "figure esthétique" au sein de l'œuvre d'art, faite d'affects et de percepts selon Deleuze et Guattari ${ }^{\mathrm{i} 2}$, et un personnage philosophique, qui se tient du côté du concept. Il prend ainsi place aux côtés de ces "génies hybrides ${ }^{13}$ » parmi lesquels Deleuze fait figurer Hölderlin, Kleist, Rimbaud, Mallarmé, Kafka, Michaux, Pessoa, ou encore Antonin Artaud - poète cité par Godard dans Je vous salue, Marie -, qui écrivait dans une lettre : "J'ai changé ior fois de personnages depuis Antonin Artaud ${ }^{\mathrm{I} 4}$.»

\section{Autoportrait(s) de la pensée}

L'idée de l'autoportrait reproduisant l'apparence physique est de plus en plus dépassée par celle d'autoportrait (fragmentaire) de la pensée, comme Godard pouvait lui-même le signifier : «Le cinéma, c'est de la métaphysique. Ça dirige le physique des gens vers la pensée ${ }^{15}$. "Même si ce qui a toujours été privilégié, au sein de l'autoportrait pictural, est la captation de l'état d'esprit, du sentiment caché de l'artiste se représentant (les autoportraits de Rembrandt sont, en cela, saisissants), le cinéma pourrait venir parfaire, par la multiplication de ses moyens esthétiques, ce sentiment de dépassement de la surface pour atteindre aux profondeurs de l'être. Cocteau, poète-cinéaste admiré, et

9. J.-L. Godard, dans Jean-Luc Godard par Jean-Luc Godard, t. 2, op. cit., p. I30.

Io. G. Deleuze, F. Guattari, Qu'est-ce que la philosophie, Minuit, "Critique», I99I, p. 6o.

II. Ibid., p. 6I.

I2. Voir ibid., p. 64.

13. Ibid., p. 65 .

I4. A. Artaud, cité dans Fl. de Mèredieu, C'était Antonin Artaud, Fayard, 2006, p. 707.

I5. J.-L. Godard, dans "Une bouleversante expérience intérieure», op. cit. 
souvent cité par Godard (la voix de François Périer-Ange Heurtebise guidant Orphée n'était pas choisie par hasard dans Soigne ta droite), disait à propos de son ultime film, Le Testament d'Orphée, qu'il était un "autoportrait d'ordre interne ${ }^{16}$ ", une

tentative d'autoportrait $[\ldots]$ qui s'attache à la ressemblance profonde et néglige cette ressemblance extérieure, qui nous documente fort mal sur un artiste lorsqu'on nous le montre dans l'exercice de ses habitudes ${ }^{17}$.

Il déclarait aussi, ce qui s'accorde aux personnages conceptuels Godard/ Oncle Jean et Godard/L'Idiot : "Ma pudeur, dans Le Testament, consiste à ne pas craindre de provoquer le rire et même à me caricaturer, comme il arrive dans le rêve ${ }^{18}$ ». Les situations dans lesquelles se dépeint Godard ne manquent pas, en effet, d'autodérision. Godard dans le rôle de cinéaste fou (car son personnage est cinéaste dans Prénom, Carmen comme dans Soigne ta droite) emprunte à ces figures du burlesque qui semblent traverser le monde dans un état d'hypnose, d'hallucination, leur propre absurdité révélant en fait celle du monde réel dans lequel elles évoluent.

Pour Godard, le cinéma est "un instrument de pensée ${ }^{19}$ » mais aussi, serait-il possible d'ajouter, un témoin des dérèglements de celle-ci. En tout état de cause s'affirme de plus en plus l'idée d'un autoportrait qui, à travers les multiples strates de sons mixés et d'images montées que permet de faire coïncider le cinéma, est capable de rendre compte de la multiplicité des lignes qui peuplent son esprit d'artiste-cinéaste / penseur-essayiste, dans une unité toujours insaisissable. Car les lignes peuvent aussi, comme le pensent Deleuze et Guattari, se faire lignes de fuite.

Histoire(s) du cinéma est une œuvre sur laquelle Godard est revenu, avec de nombreux repentirs semblables à ceux d'un peintre, pendant dix ans, de I988 à 1998 ... Seuls quelques moments de ce travail, finalement toujours inachevé (le cinéaste en a aussi proposé plus récemment une version écourtée) peuvent être évoqués ici, où la question de l'autoportrait se manifeste plus directement, même si l'œuvre en son ensemble peut être considérée comme un autoportrait mental de l'auteur. Cette réalisation est composée de multiples fragments empruntés aux films de Godard, mais aussi à l'histoire du cinéma, à celle de la peinture et à l'Histoire du $\mathrm{xx}^{\mathrm{e}}$ siècle, dans une organisation d'une infinie complexité, où tous les flux entrent en corrélation au sein d'une matière vidéo qui métamorphose les images filmiques, qu'elles soient documentaires ou fictionnelles.

I6. J. Cocteau, Du cinématographe, Le Rocher, Monaco, 2003, p. 240.

17. Ibid., p. 237.

18. Ibid., p. 239.

19. J.-L. Godard, dans Jean-Luc Godard par Jean-Luc Godard, t. 2, op. cit., p. 294. 
Lors d'une première apparition de Godard au sein d'Histoire(s) du cinéma, celui-ci se montre installé derrière le clavier d'une machine à écrire. Ce choix n'est évidemment pas le fruit du hasard, et a déjà été fait par Godard longtemps auparavant, dès le court métrage de ses débuts Charlotte et son Jules. Ici, la machine - qui n'est pas encore un ordinateur - est juste passée de mécanique à électrique. Fait important, puisque Godard déclenche des sortes de salves très sonores avec celle-ci, devant un micro que l'on perçoit entrant dans le champ visuel. Des images naissent en surimpression sur le visage du cinéaste, et se superposent ainsi les strates de l'écriture, du son, de l'image de soi et des images des extraits qui naissent dans les rafales de la machine à écrire. Comme si, tout à coup, se conjuguaient la caméra-œil de Vertov dans Loin du Vietnam, la "caméra-stylo" pensée par Alexandre Astruc, la "cinécriture» pensée par Agnès Varda, et la caméra-radio de Prénom, Carmen. Une autre apparition de Godard se fait, alors qu'il se tient debout près de sa bibliothèque, le visage à hauteur d'un rayonnage de livres - de tous ces ouvrages dont les titres et les fragments constituent les cartons de ses films des années 1990, et nourrissent à l'infini son imaginaire. Dans le clignotement d'une forme d'équivalent vidéo d'une fermeture-ouverture à l'iris très accélérée, flashes de mémoire et battements de cœur, des images d'archives et de fiction surgissent et s'effacent, la machine à écrire-mitrailleuse continuant de scander le montage-mixage rythmique des images et des sons. Dans un troisième moment, le visage de Godard devient littéralement "transparent» dans la surimpression : et ce sont les images du Mépris qui traversent l'esprit du cinéaste. Godard en personnage d'assistant, sur le tournage d'une imaginaire Odyssée réalisée par Fritz Lang en 1963, peuple le visage de Godard en historien du cinéma de 1988 , dans une véritable coalescence d'actuel et de virtuel, selon la terminologie de Deleuze dans sa définition de l'ImageTemps... Un «autoportrait de la pensée» fait en effet que le fragmentaire ne se développe plus seulement dans l'espace, mais aussi dans le temps.

\section{Autoportrait(s) cristallin(s)}

Dès les trois premières minutes de $J L G / J L G$ (autoportrait de décembre) se produit une remarquable "cristallisation" - comme il s'en crée selon Deleuze dans le "régime cristallin de l'image», tel qu'il le définit dans la continuité des travaux d'Henri Bergson - faite d' "opsignes» et de "sonsignes" qui donnent forme matérielle au concept d'Image-Temps, et donc aux actualisations de virtualités qu'il induit : 
- L'ombre de Godard à la caméra se porte sur la photo en négatif de Godard enfant. Tandis que sa voix, "à bout de souffle" pourrait-on dire sous forme de clin d'œil, se fait spectrale, comme revenue d'outre-tombe...

- Des cristaux de temps se forment dans les vitres, reflets, écran de contrôle de la caméra vidéo, écran de télévision, fenêtre dans laquelle s'entraperçoit l'image de Godard en profondeur de champ.

- Godard revient au premier plan, sortant des «nappes de passé» pour, en une "pointe de présent", commencer à écrire le texte de son très court métrage Je vous salue Sarajevo, dans lequel il oppose la culture à l'art et à l'art de vivre...

- Le début du film est par ailleurs traversé par les fulgurances des "paysages d'enfance» du lac Léman, déchiré par la violence des sons : vagues, mouettes, aboiements de chiens, sonneries de téléphone, et habité par la mémoire sonore d'un film de Roger Leenhardt concernant l'enfance, dans un milieu protestant cher à Godard : Les Dernières vacances (1948).

Un autoportrait cristallin se constitue donc, composé de multiples facettes temporelles, auxquelles renvoie le carton-citation «SEIN UND ZEIT» (Etre et Temps), célèbre titre de Martin Heidegger ( $L L G / J L G$ est d'ailleurs, pour Philippe Sollers, un "film sur l'être-làa ${ }^{20}$ ). La barre qui sépare les JLG est aussi présente, comme l'indique Godard, "pour montrer JLG dans le miroir, ce qui se fait dans l'autoportrait ${ }^{21}$ », et le miroir est pour Deleuze la figure primordiale du passage entre le virtuel et l'actuel.

\section{Autoportrait(s) complexe(s)}

La toute fin d'Histoire(s) du cinéma, qui est donc celle de l'ultime épisode (4B) intitulé "Les Signes parmi nous" (phrase empruntée au titre d'un roman de Charles-Ferdinand Ramuz, source suisse et, même lémanique, très importante pour Godard), permet de conclure sur cette idée d'un autoportrait fragmentaire dépassé par celle d'un autoportrait complexe. Ces signes qu'il s'agit de percevoir coïncident avec cette très belle phrase que Manoel de Oliveira a prononcée pour définir le cinéma de Godard : "C'est d'ailleurs ce que j'aime en général au cinéma : une saturation de signes magnifiques qui baignent dans la lumière de leur absence d'explication ${ }^{22}$."

Dans ce dernier moment, la voix off de Godard prononce une phrase de Samuel Coleridge, qui avait déjà été abordée par Jorge Luis Borges dans l'une

20. Ph. Sollers, "Un cinéma de l'être-là", Cahiers du cinéma, n 489, mars 1995, p. 39.

21. J.-L. Godard, dans Jean-Luc Godard par Jean-Luc Godard, t. 2, op. cit., p. 306.

22. M. de Oliveira, dans "Godard et Oliveira sortent ensemble", Jean-Luc Godard par Jean-Luc Godard, t. 2, op. cit., p. 270. 
de ses enquêtes : «Si un homme traversait le Paradis en songe, qu'il reçût une fleur comme preuve de son passage, et qu'à son réveil, il trouvât cette fleur dans ses mains... Que dire alors?», prolongée par cette réponse : "J'étais cet homme»... À l'image est alors vu le visage de Godard en gros plan qui fusionne, dans une unité nouvelle, avec le tableau de Francis Bacon, Étude pour un portrait de Van Gogh I (1956), réinterprétation de la toile de Van Gogh - détruite pendant la Seconde Guerre mondiale : Autoportrait sur la route de Tarascon (I888)...

Comme si l'autoportrait de Godard, dont le visage semble se remplir de la peinture de la toile par une surimpression vidéo, contenait en lui l'autoportrait de Van Gogh dans lequel s'était lui-même reconnu Bacon, et se chargeait encore de la poésie de Coleridge, dans laquelle Borges avait puisé cette phrase, qui s'accorde à présent à la traversée par Godard de l'Histoire du cinéma et de sa propre existence...

Voici bien ce qui fait coïncider l'autoportrait cinématographique selon Godard, et les propos que Deleuze consacre à ce cinéaste plongé dans une «solitude absolue ${ }^{23}$ » mais une "solitude extraordinairement peuplée $e^{24}$ », "une solitude multiple, créatrice ${ }^{25}$ ", un "bégaiement créateur [...] qui fait de Godard une force ${ }^{26} »$.

Il est permis de penser que toutes ces multiplicités dépassent en fait le fragmentaire, puisque les autoportraits de Godard créent finalement des relations infinies entre les lignes qui les composent. Forme d'organisation complexe, spatiale et temporelle, où les deux $J L G$, personnages conceptuels situés de part et d'autre du miroir, entrent cependant en interaction avec la troisième personne que constitue l'homme Jean-Luc Godard, dans un champ d'intensité nouveau, une triade dynamique.

23. G. Deleuze, Pourparlers, Minuit, 1990, p. 55 .

24. Ibid.

25. Ibid.

26. Ibid., p. 56. 